\title{
Energy saving in the street lighting control system-a new approach based on the EN-15232 standard
}

\author{
Andrzej Ożadowicz • Jakub Grela
}

Received: 25 March 2015 / Accepted: 24 August 2016/Published online: 30 August 2016

(C) The Author(s) 2016. This article is published with open access at Springerlink.com

\begin{abstract}
The street lighting is one of major components in total energy consumption in cities. The paper is focused on a concept of street lamp control systems and function organization with remote monitoring, to reduce maintenance costs and energy consumption. A new approach to the definition of functional strategy organization for outdoor lighting systems is introduced in the paper. Proposed functional strategies are based on four efficiency classes of building automation and control systems (BACS) defined in the EN 15323 standard. They have been formulated, analysed and eventually implemented and verified in real experiment street lighting installation. This outdoor lighting system, designed by the authors, based on LonWorks (the ISO/IEC EN 14908) platform with a power line communication aimed to control high-pressure sodium lamps. The street lamps are integrated nodes of a building management system (BMS). The results of experimental tests for the proposed functional strategies, implemented with various control scenarios, show that they provide a great potential in reducing energy consumption by street lighting installations. In particular, the energy use can be reduced even by $45 \%$ in comparison to conventional street lighting system, especially without the use of monitoring and control.
\end{abstract}

\footnotetext{
A. Ożadowicz $(\bowtie) \cdot J$. Grela

AGH University of Science and Technology, al. Mickiewicza 30, 30-059 Krakow, Poland

e-mail: ozadow@agh.edu.pl
}

Keywords The EN-15232 standard LonWorks . Power management - Street lighting - Energy efficiency. Building automation. Power line communication

\section{Introduction}

The street and outdoor lighting installations provide citizens with safe roads, stylish and inviting public areas and enhance the security in their homes, businesses and city centres. They are very important elements of infrastructure in cities around the world. Unfortunately, according to different reports, the street lighting is one of the biggest electrical energy consumers, accounting for about $40 \%$ of the total energy consumption in cities (The European PPP Expertise Centre (EPEC) 2010; European Commission 2013; Mohamed 2013). This shows that they are costly and contribute considerably to environment pollution. The production of electricity needed to power the street lighting systems significantly adds to carbon dioxide emissions ( $\mathrm{CO} 2$, greenhouse gas) and nuclear dust. The costs of the electrical energy together with the environmental factors encourage municipalities to implement solutions to measure, analyse and reduce energy consumption in order to reduce spending and decrease maintenance costs (SungKwan Cho and Dhingra 2008; Li-jun et al. 2011). All round the world, especially in cities, monitored light installations of public spaces are implemented and controlled manually or remotely. These can be commercial technical solutions and research pilot ones. The leaders are the 
USA, the European Union's countries and China. In many of them, the total number of lighting points is calculated in the tens of thousands, for instance: Oslo, Norway-65,000 lamps; Varna, Bulgaria-1000 lamps; Amsterdam, Netherlands-2300 lamps; Dublin, Ireland-5000 lamps etc. (E-Street Project Grant Team 2008; Echelon Corp. 2011). In a longer perspective, an integration of the many control and city infrastructure maintenance systems, including street lights, is expected to build complete, integrated networks of data and information exchange, integrated monitoring and control-so-called the smart cities systems. This operation will allow effective energy and media management, together with energy demand management (DemandResponse concept) and services for prosumers (Palensky and Dietrich 2011; Noga et al. 2013; Oliveira-Lima et al. 2014; Tascikaraoglu et al. 2014). So far, research and tests of the smart lighting systems have been carried out, to compare them and their functionalities with the previously used technical solutions, especially in the quality and user comfort areas, as well as in relation to energy and maintenance costs savings. Another investigation concerns the exploration and development of lighting control algorithms. Two main approaches are analysed: static and dynamic. The first of them is based on the constant settings. The parameters like (i) the type and power level of light sources; (ii) the on/off time periods, according to the calendar; (iii) changing the length of day and (iv) light sensor signal are set to the lighting control system. The second approach — dynamic algorithms, offer more possibilities of control, optimization and adjustment of the lighting. But it requires significantly more infrastructure elements and may have different functional development levels. The simplest solutions allow to change light intensity at certain periods of time (e.g. at different hours of the night). This results in different time-action lighting profiles, providing additional energy savings and increasing the lifetime of the lamps (Mohamed 2013; Pizzuti et al. 2013; Wojnicki et al. 2014). This paper is focused on implementation of the distributed building automation and control systems (BACS), based on the international standards, in the street lighting field. They provide the possibility to control the lamps individually or in a group, implement both the simple and advanced control algorithms focused on energy saving, $\mathrm{CO} 2$ reduction and tools to monitor, manage and run diagnostics of the lamps (Arnold and Barth 2012; Leccese 2013; Colmenar-Santos et al. 2013). It is possible to integrate the outdoor and street lighting monitoring and control data with the building management system (BMS) in the building. In more functionally advanced systems, various types of proper sensors and other infrastructure subsystems are integrated. These include, for example: weather stations, motion/presence and light intensity sensors, elements of wireless and mobile phones networks etc. (Novak and Gerstinger 2010; Müllner and Riener 2011; Novak et al. 2014; Todorović and Samardžija 2016). Structure of research installation is presented and explained in "The street lighting system-experimental installation" section. In this paper, we propose a new approach to formulation of control system functional strategies, based on the BACS efficiency classes. A possibility to use the EN-15232 guidelines in a new field e.g. organisation of control systems for street lighting installation has been investigated. This study, described in the paper, focuses on the impact of the control systems on the outdoor lighting energy consumption reduction. This can be achieved by technical, organisational or behavioural changes. Application of the BACS efficiency classes allows to categorize different, simple and advanced control systems and assess their impact on street light energy use in general (Schiavon and Melikov 2009; EN 15232 Standard 2012; Schönenberger 2015). In our opinion, this approach and the proposed system organization are universal and useful for integrated automation system designers and integrators, creating an easier path to energy efficiency improvements. Assumptions for the proposed scenarios, implementation process and results are presented in the "Implementation and verification of control scenarios" section.

\section{The street lighting system-experimental installation}

Technical parameters and functions of an experimental street lighting system are presented in this section. Their analysis and verification have been the first stage of our research works. A research installation of intelligent public space lighting is located at one of the car parks of a university campus - Fig. 1.

Besides the standard functionalities of classic street lighting systems, associated with lighting a particular space, the experimental installation has advanced monitoring and control ones. A control system is based on the ISO/IEC EN 14908 BACS standard (LonWorks) 
Fig. 1 The car park real photo with the experimental street lighting installation

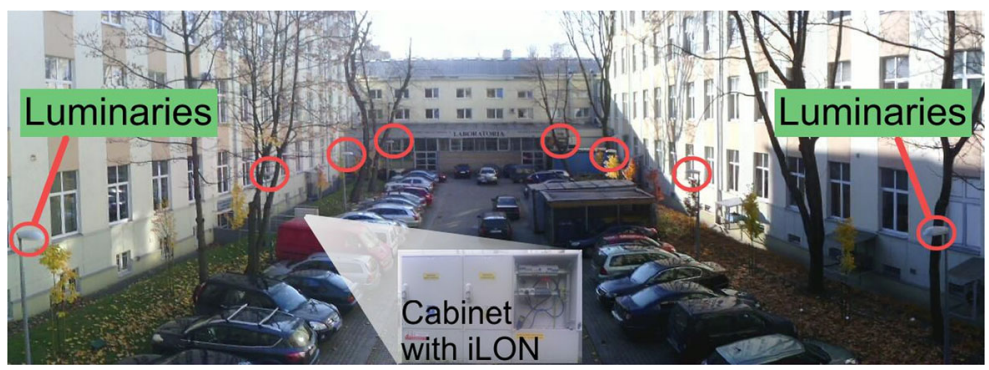

with a power line communication (PLC) on the field level, to control each of a lamp. The PLC technology is an excellent solution widely studied and analysed by researchers, even in areas characterized by strict requirements, such as industries. Its reliability has been investigated and verified taking into consideration different aspects, in order to optimize the use and the performance depending on the specific environment (Sutterlin and Downey 1998; Collotta and Tirrito 2014). In our experiment, the PLC technology is used as one of the communication channels. A schematic diagram of the system is shown in Fig. 2.

The lamps as BACS nodes

Our experiment is carried out on the installation consisting of eight modern road lighting luminaires with the high pressure sodium lamps (HPS 70W). In each of the luminaires, there are three additional modules: LonWorks node module, an electronically adjustable smart ballast and an interface filter module (Fig. 3). The applied electronically controlled smart ballast is a source of power for HPS lamps. It ensures optimal lamp use parameters throughout the period of its operation, extending the life of the lamp and reducing energy consumption compared to conventional ballasts (Hsieh and Lin 2011; Moo et al. 2011; Gutierrez-Escolar et al. 2016). The mentioned LonWorks network node module is equipped with a Neuron Chip processor 31xx series and Power Line transceiver (Sutterlin and Downey 1998). This device is the interface between the ballast module and the data transmission medium (in this system, its supply network-Power Line Communications). The lamps with these devices are autonomous control nodes in the distributed automation LonWorks network (Marcos Alonso et al. 2000; Onat et al. 2011; Li-jun et al. 2011; Dragičević et al. 2014; Oliveira-Lima et al. 2014). A block diagram with connections between the modules in the lamp is shown in Fig. 3.

Additionally, in each luminary, a filter module has been implemented to ensure the high reliability of the data stream received by the node modules or sent to other system components via a transmission medium PLC. It eliminates data read errors caused by noises and interference, appearing in the transmission medium (Onat et al. 2011). The luminaires fitted with the
Fig. 2 A schematic diagram of a research street lighting installation

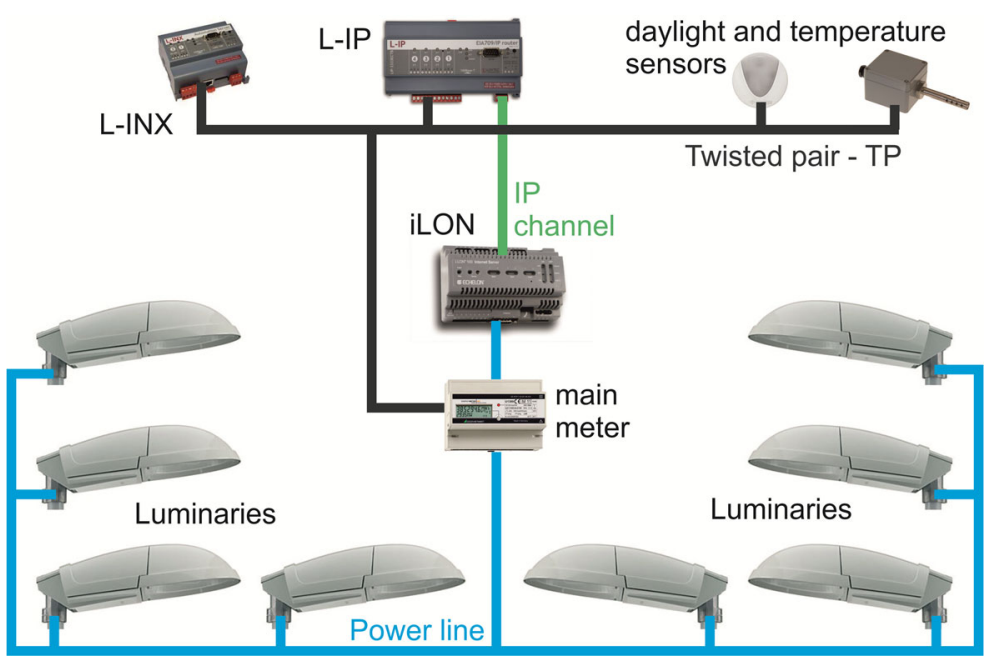




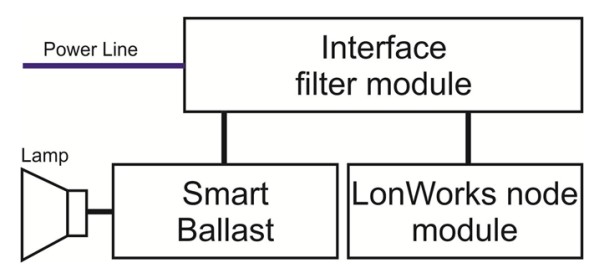

Fig. 3 The block diagram of the connections between modules in the lamp

mentioned devices, use the advantages offered by the LonWorks technology and provide remote monitoring and control level of the load lamp (the on/off function and adjusting the level of illumination), monitoring of the supply voltage for each of the lamp, measurement of energy consumption, information about the number of work hours and other work parameters. All of the mentioned data could be sent to the BMS and integrated within the BACS.

\section{BACS infrastructure}

The integrated system of street lighting includes a server module i.LON (Figs. 1 and 2). In our experimental installation, it is a router for the LonWorks standard communication between two kinds of the transmission medium: Power Line and IP network (Internet). Routing is realized by tunnelling LonTalk protocol telegrams in IP standard frames. The i.LON module also works as a Web server, with additional control functions for example, in our studies, a scheduling function has been used see the "Control scenarios-descriptions and assumptions"section (Li-jun et al. 2011). Another component of the system is a L-IP router (Fig. 2). It receives or transmits data packets to the i.LON router via IP networks and IP-852 technology as well as enables connection to the part of the system realized with another transmission medium - twisted pair (TP) (Soucek and Sauter 2004). This allows to integrate the outdoor lighting control system with the BMS in a university campus building near the car park. In this particular installation the daylight level sensor has been connected and integrated this way. The L-IP router and some parts of the infrastructure of the system are placed in cabinets in the mentioned building (Noga et al. 2012). The lamp control system interoperates with a daylight sensor and a temperature sensor (Fernandes et al. 2014). They systematically provide information on the current level of ambient light intensity or the outside temperature to all devices running on the LonWorks technology. A diagram of network connections between described automation and monitoring modules, along with data channels, is shown in Fig. 4. It presents the view of the experimental street lamp control network in the Lon Maker package - software for integrators.

An automation server L-INX from the BMS has been implemented in the street lighting system as well (Fig. 2). In this experimental installation the L-INX module is used to provide monitoring and visualization of the system and lamp status. The visualisation is a key part of a technical building management (TBM) system. In our experiment, it has been used to monitor and control the research street lighting system installed at the mentioned car park of the university. Designed by us, the visualization panels are available by a standard Web browser, from anywhere (with access to the Internet). Starting them and setting the visualization options
Fig. 4 Project of the street lighting installation in the LonMaker package, with nodes and data channels - different kinds of the communication media

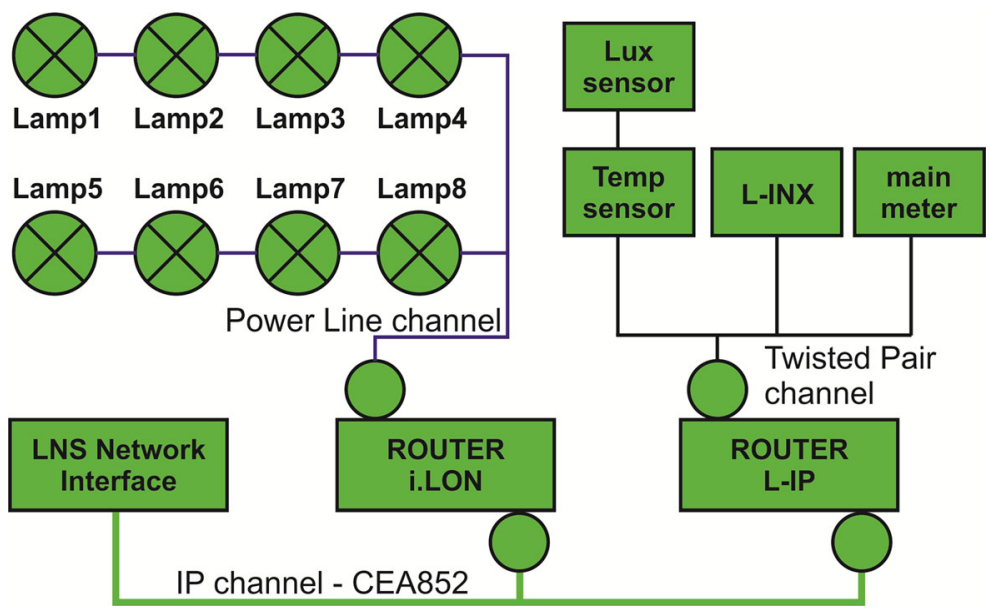


require proper authorization and could be encrypted. The visualization allows to remotely monitor and manage the state of the entire street lighting system, the groups of the lamps and the state of each lamp separately. Figure 5 shows one of the graphical panels.

The lamp status monitoring provides information about on/off states; light intensity level (low_ $\%$, medium- $60 \%$, high- $80 \%$ ); the voltage at the contacts of the light source mounted in the luminary, the total electrical energy consumption, amount of working hours, temperature of the ballast and state of the alarm signals. The visualization has also reporting capabilities about errors or hazards that may arise during system operation. They are parts of the mentioned TBM system as well. For instance, Fig. 5 shows a situation when the lamp is loaded to the maximum and the voltage on the light source is 139 VAC. This value is too high and it reports that the life-end of the HPS lamp is approaching. The system informs the user about this fact, not only by changing the colour of an icon (in Fig. 5-red) but also by sending an e-mail to the service, with information about the need to take appropriate action. This feature is an additional value of our TBM and control system, helpful in maintenance services.

\section{Electrical energy measurement instrumentation}

There is one electrical energy meter implemented in the experimental street lighting installation (see Fig. 2). A main meter measures total energy consumption for all of the eight lamps. The meter complies with the MID directive which is valid in $\mathrm{Eu}-$ rope-the Measuring Instrument Directive 2004/22/
EC (MID). The energy meter accuracy class B, roughly corresponds to class 1 in the well-known meter standards. This meter has been manufactured according to the regulations of the following standards: the EN 50470-1, the EN 50470-3 and the EN 62053-23. As has been mentioned, each lamp - the LonWorks network node, has its own built-in energy meter. The measurement data from each of the lamps have been sent as network variables. They have been collected, summed and compared with the main energy meter indications. Deviations of the measurements have been negligible and correspond to the class B. In this respect, we decided to calculate energy consumption for each of the lamps, using the main energy meter indications.

\section{Implementation and verification of control scenarios}

The street lighting system with the mentioned devices and functions is an object of our research and real experiments. Ongoing research works are aimed at analysing the possibility of reducing the energy consumption in the outdoor lighting installation, by functions of the distributed building automation systems (LonWorks) and to determine the effect of various functional organization strategies and control algorithms, implemented in the networked control system, on the energy consumption in the lighting system (BurgosPayan et al. 2012; Mohamed 2013; Pizzuti et al. 2013). Therefore, in our opinion, it makes no sense to compare our outdoor lighting installation with other existing street lighting control systems. We have not
Fig. 5 Screenshot of the operating status for a single lamp in the street lighting system-an element of the TBM system

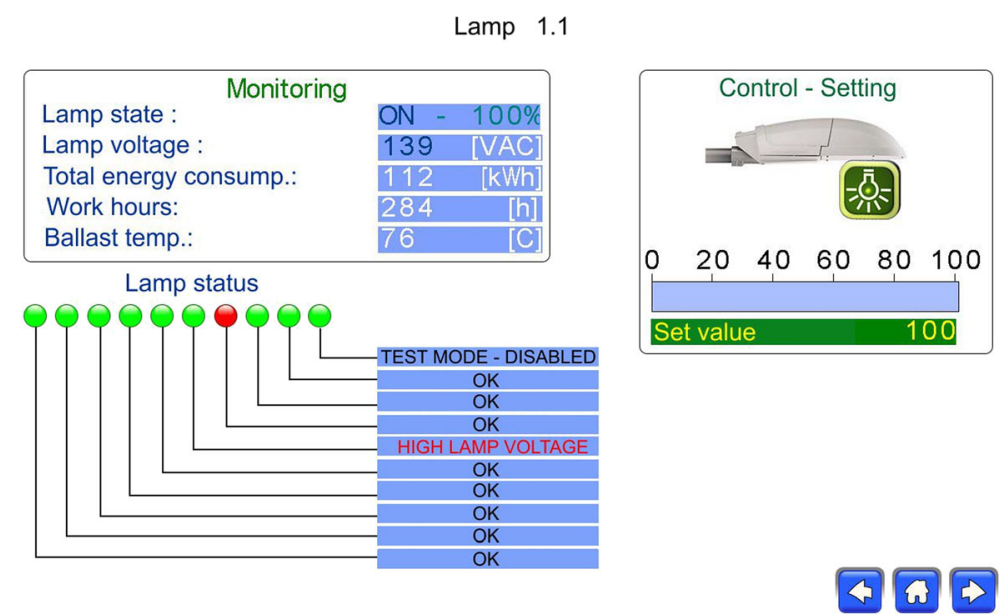


been focused on the differences between the devices and technologies used in street lighting installations. Our concept, presented in this paper, is universal and could be used to evaluate the organization, functional strategies of the street lighting control systems and their impact on the energy consumption for installations with all kinds of lamps (HPS, LEDs etc.). Today, road and street lighting commonly uses high-intensity discharge (HID) lamps. The HID lamp family includes HPS (Todorović and Samardžija 2016). The second stage of our research works has been energy consumption comparison between lamps equipped with two different kinds of ballasts. In the experiment, by default, each of the luminaire has been equipped with high-pressure sodium source (HPS 70W) and appropriate modules, including an electromagnetic ballast. The consumption of electrical energy by a single lamp in average daily cycle, assuming $15 \mathrm{~h}$ of operation, was $1.54 \mathrm{kWh}$. Following that, all standard manufacturer's modules had been replaced by electronic modules described in "The lamp as BACS nodes" section. As a result, energy consumption in an average daily cycle was $1.3 \mathrm{kWh}$. A comparison of the energy consumption is shown in Fig. 6. A data acquisition period for these measurements is 105 days.

It should be emphasized that the lamp offered by the manufacturer did not have dimming and/or energy consumption reducing capabilities. The measurements were carried out for the maximum load for the lamps and their Smart Ballasts (SungKwan Cho and Dhingra 2008; Burgos-Payan et al. 2012; Ożadowicz and Grela 2014; Fernandes et al. 2014).
Functional strategies - implementation of the EN-15232 standard

In the next stage of our research works, some different functional organization strategies of the control system have been proposed in a new, original approach. Since the street lighting control system based on the LonWorks technology — one of the BACS standards, the proposed strategies have been formulated in an analogy to efficiency classes in building automation control systems (BACS), described in the European Standard EN 15232 "Energy performance of buildings-Impact of Building Automation, Controls and Building Management" (EN 15232 Standard 2012). This standard concerns the impact of the BACS and the TBM on energy performance and energy use in buildings. There are four BACS efficiency classes described in this document: (i) the class A corresponds to the BACS with very high impact on energy consumption, (ii) the class B-the BACS with high impact on energy use, (iii) the class C - the BACS with low impact on energy consumption and (iv) class D-the BACS without any impact on the energy consumption in buildings (Djuric and Novakovic 2010; Schönenberger 2015). Considering this, and taking into account the assumptions for the classes as well as the functions available in the street lighting control system, in our experiment, we propose four different functional strategies (EN 15232 Standard 2012):

- The street lighting installation with the standard lamps (the electromagnetic ballast), not offering any control options (only basic switch on/off all
Fig. 6 The electrical energy consumption for the standard lamp modules and new ones (Smart Ballast)

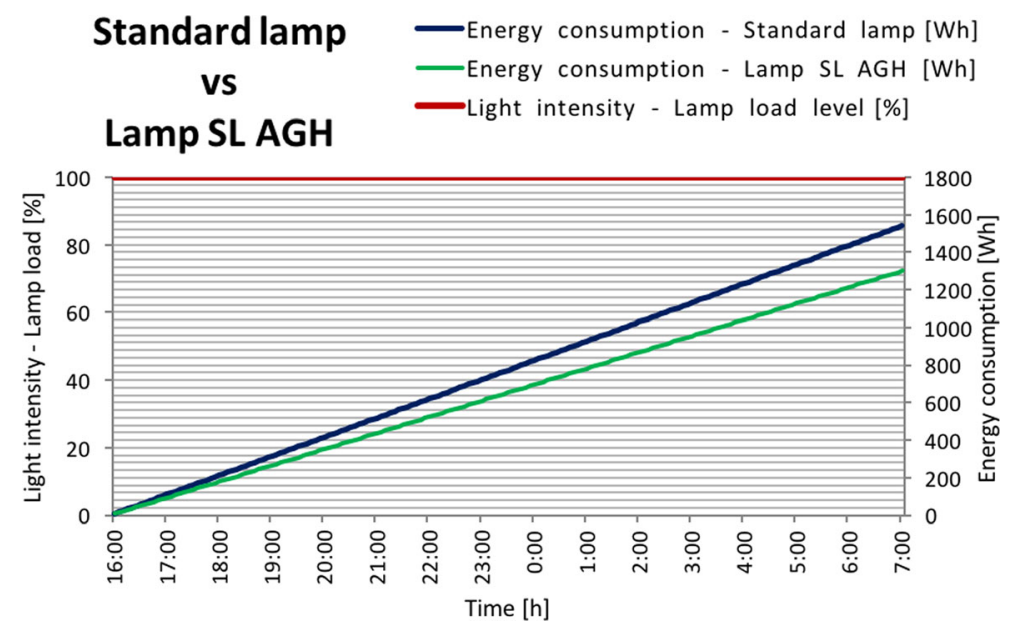


the lamps simultaneously). This strategy corresponds to class D.

- The street lighting installation consisting of the lamps equipped with the modern electronic modules providing a reduction of energy consumption, preserving the light intensity like the standard lamp, extending the life of the lamps, without advanced functions related to settings of lighting intensity and technical management of the installation. There is possibility to switch the lamps on/off independently of each other. The strategy corresponds to class $\mathrm{C}$ (see Fig. 6).

- The street lighting installation with the elements and functions from previous class $\mathrm{C}$ strategy and additional advanced control and selected technical management functions of the installation: automated control with time schedules implemented in controller or automation server module, to adapt to different scenarios of usage the lighting installation and to reduce energy consumption. It is possible to implement individual and group control of the lamps. This strategy corresponds to class B.

- The street lighting installation with elements and functions from previous class B strategy and the advanced functions of the control and technical management. The control strategy takes into account mentioned time schedules, but it is focused on maximum daylight usage with automatic adaptation to external condition changes (daylight levelprovided by Lux sensor integrated in the network) and adjusting the light level depending on occupation. The daylight changes do not only depend on time of the day but also on local weather conditions. This fact has been taken into consideration by implementation of proper sensors (e.g. Lux sensor, etc.). This strategy is based on full integration between the street lighting installation and the BMS. This approach allows to implement various elements and functions of the building control and monitoring system, with an interoperability between them. The described strategy corresponds to class A.

For the classes B and A, additionally to the mentioned advanced control functions and the interoperability of the control data, some of the TBM functions have been implemented - for instance: (i) lamps and control device failure detection and support in their fixing and (ii) monitoring of the installation work parameters (voltage level, ballast temperature, run hours) with alarms detection. The outdoor lighting system integration with the BMS provides synergy between various applications, improving comfort, safety and energy savings in the lighting installation. In this context the BACS functions, integration with the BMS and building automation systems, are important enablers of a new possibilities in control, monitoring and energy savings in the street lighting installations. In our study the functional strategies and energy savings provided by them are the main research question.

Control scenarios - description and assumptions

To implement and verify the functional strategies for the classes B and A, proposed in the "Functional strategies - implementation of the EN-15232 standard" section, we decided to test them with several scenarios of control-for various time schedules and settings of parameters associated with the level of light intensity. The approach was based on similar works conducted in other research centres (Chung et al. 2005; Alzubaidi and Soori 2012; Omar et al. 2013; Pizzuti et al. 2013). The master device controlling the lamps in these studies was the i.LON module, described in the "BACS infrastracture" section. With its scheduling functions for the devices integrated in a single project of the LonWorks control network, some scenarios have been prepared and verified practically and numerically. The first scenario (scenario 1) provides a relatively high level of comfort in lighting of the concerned parking area and creates possibility to find good time intervals to reduce the level of light intensity, with the assumption that it is unacceptable to switch the lamps off during the night. Day profiles of a lamp load, corresponding to this scenario are presented in Figs. 7 and 8 (red solid line). The second scenario (scenario 2) is a modification of the first one. Based on the observations, some modifications have been implemented in the levels of light intensity for given working periods of the lamps. These changes provide levels of light intensity more suited to the different types of usage of the mentioned parking area, with the assumption that it is unacceptable to switch the lamps off during the night. Day profiles of a lamp load, corresponding to this scenario are presented in Figs. 9 and 10 (red line). The third scenario (scenario 3) was created as a modification of the second one, with only minor changes in the level of light intensity settings as well as the ability to switch a few lamps off in the time period identified as "lack of use of the parking". Day profiles of a lamp load, 
Fig. 7 Scenario 1 class

B - measurements and results of energy consumption
Scenario No 1

- Light intensity - Lamp load level [\%]

-Energy consumption - nominal [Wh]

-Energy consumption - scenario [Wh]

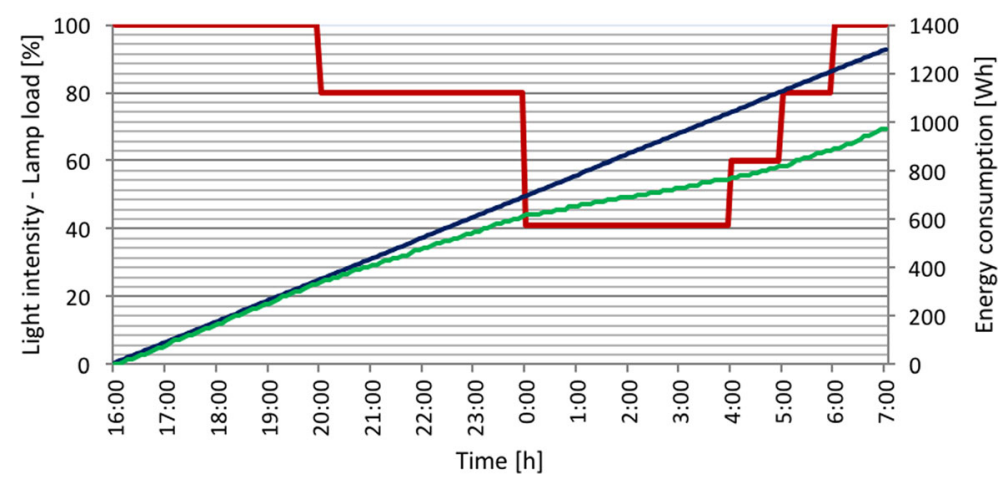

corresponding to this scenario are presented in Figs. 11 and 12 (red line). All proposed scenarios have been accepted by the technical department of the university as well as car park users and provide sufficient lighting conditions. These three scenarios have been implemented in the street lighting control system devices in respect of the mentioned functional strategies corresponded to the BACS energy efficiency classes B and A. This way, we would like to show differences in energy consumption between these both strategies (different strategies verified with the same group of three scenarios) and eventually between them and other two-corresponded to $\mathrm{C}$ and $\mathrm{D}$ classes. Energy consumption for the class D and class $C$ have been measured in the second stage of our research works and described already at the beginning of the "Implementation and verification of control scenarios" section.
Experimentation and results

Experiments and tests have been the another stage of our research works and have been carried out on real data, measured in the street lighting system described in the "The street lighting system-experimental installation" section.. The results of observations and measurements of the energy consumption in the lighting system for the mentioned functional strategies, are presented in the form of graphs. For each of them, the red solid line shows the change in the level of the lamp load (light intensity values in \%). The green solid line-energy consumption of the lamp for a given scenario [Wh]. The dark blue solid line represents the energy consumption of the lamp equipped with electronic systems described earlier, but in the absence of the lamp load (light intensity) level regulation [Wh]. The average daily
Fig. 8 Scenario 1 class A - measurements and results of energy consumption

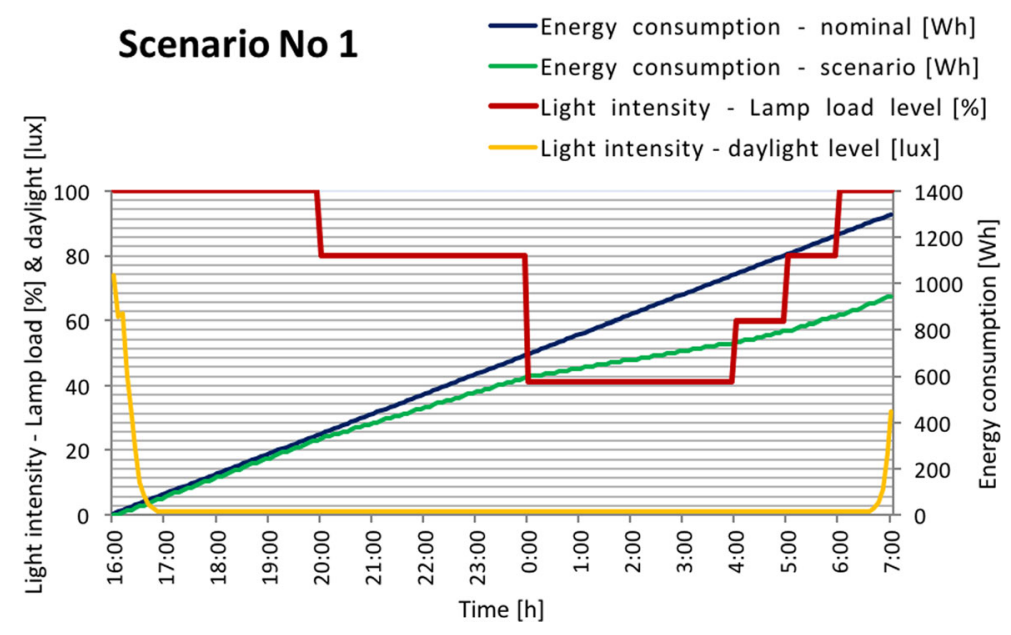


Fig. 9 Scenario 2 class

$\mathrm{B}$ - measurements and results of energy consumption
Scenario No 2 -Energy consumption - nominal [Wh]

-Energy consumption - scenario [Wh]

-Light intensity - Lamp load level [\%]

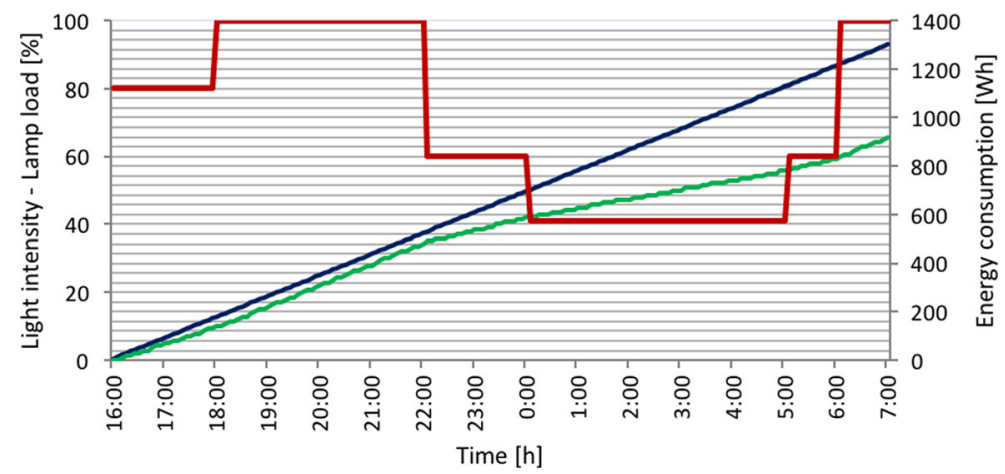

consumption of the electrical energy for a single lamp is presented on the graphs. For each the lamp a 15-h work cycle has been considered. The first two graphs (Figs. 7 and 8) refer to measurements for the Scenario 1 and two functional strategies corresponded to the B and A BACS efficiency classes. The average daily consumption of electrical energy for the single lamp for class B is $0.97 \mathrm{kWh}$ and for class $\mathrm{A}$ is $0.94 \mathrm{kWh}$. A data acquisition period for these measurements is 52 days.

The next two graphs (Figs. 9 and 10) show measurements for the scenario 2 and two functional strategies corresponded to the B and A BACS efficiency classes. The average daily consumption of electrical energy for the single lamp for class B is $0.92 \mathrm{kWh}$ and for class A is $0.89 \mathrm{kWh}$. A data acquisition period for these measurements is 57 days.

The last two graphs (Figs. 11 and 12) present measurements for the scenario 3 and two functional strategies corresponded to the B and A BACS efficiency classes. The average daily consumption of electrical energy for the single lamp for Class B is $0.87 \mathrm{kWh}$ and for class $\mathrm{A}$ is $0.85 \mathrm{kWh}$. A data acquisition period for these measurements is 66 days.

From these three comparisons based on different control scenarios, it is clear that in general, the functional strategy corresponded to the class A provide energy savings, compared with the class B. The savings are not very significant, but it is important to take into account that the presented data and results concern only 1-day periods.

The measurements and calculations of the street lighting system energy consumption for longer periods have been the last part of our study. We decided to present results for a 1-year period. The results concern whole street lighting installation (8 lamps). Bearing in mind all technical and environmental conditions and
Fig. 10 Scenario 2 class A - measurements and results of energy consumption

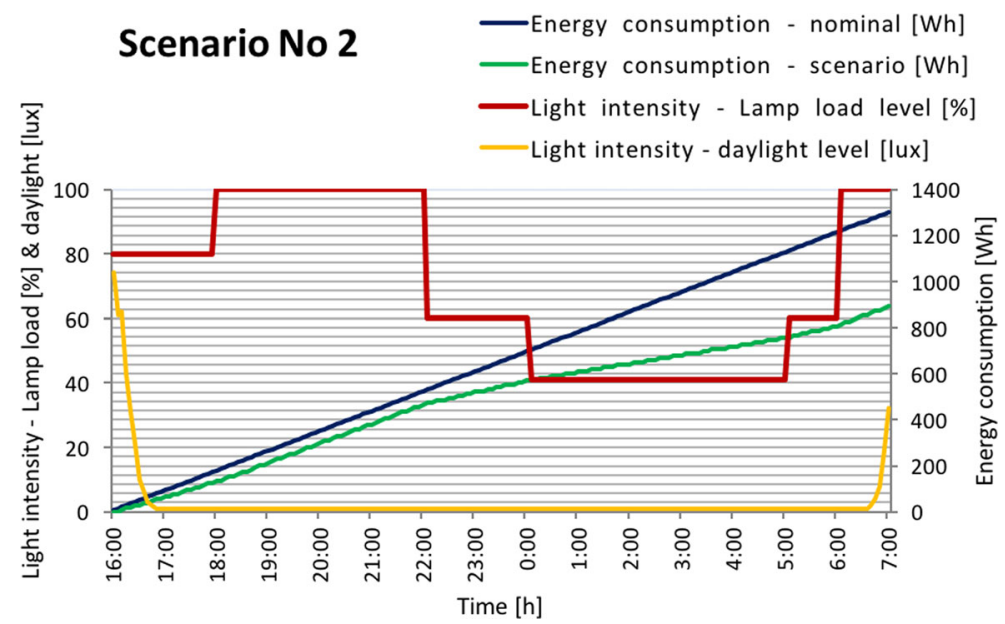


Fig. 11 Scenario 3 class

$\mathrm{B}$ - measurements and results of energy consumption
Scenario No 3

-Energy consumption - nominal [Wh]

-Energy consumption - scenario [Wh]

—Light intensity - Lamp load level [\%]

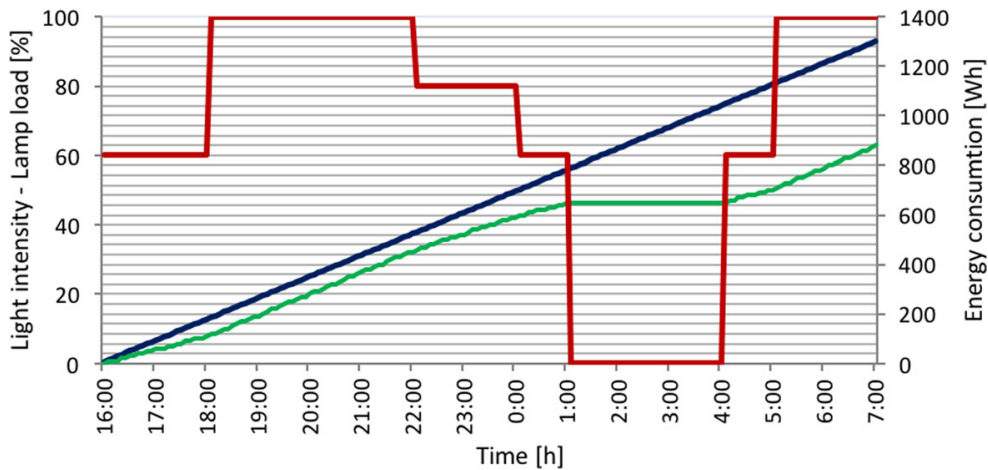

their changes during the year, for our calculations and analysis, we assumed:

- The results concern whole street lighting installation (8 lamps).

- All control scenarios are constant-settings are the same throughout the year.

- For functional strategy corresponded to the class A (with daylight level sensor) switching on/off the lamps also depends on astronomical calendar for the location of the street lighting system - sunsets and sunrises.

- For the classes D and C-without control scenarios - the overall work time for each of the lamp in the lighting system is $4500 \mathrm{~h}$.

The results of the mentioned measurements and calculations are presented in Fig. 13.
Results discussion

The studies and research presented in the paper show that using modern technologies and the BACS elements to reduce lighting energy consumption and manage power demand in the public spaces is reasonable and economically viable. Integration of the lamp installation with advanced controllers as well as additional sensors in the distributed automation networks and the BACS is the crucial element of our approach. The proposed functional strategy corresponded to the class B assumptions (Figs. 7, 9, and 11) causes the street lighting system to have a much lower energy consumption compared to installations made with standard lamps (Fig. 6), which do not have functions of scheduling and regulating the light intensity level. The reduction of electrical energy consumption is approximately by $41 \%$. Then, functional strategy made consistent with the class $\mathrm{A}$ of the
Fig. 12 Scenario 3 class A - measurements and results of energy consumption

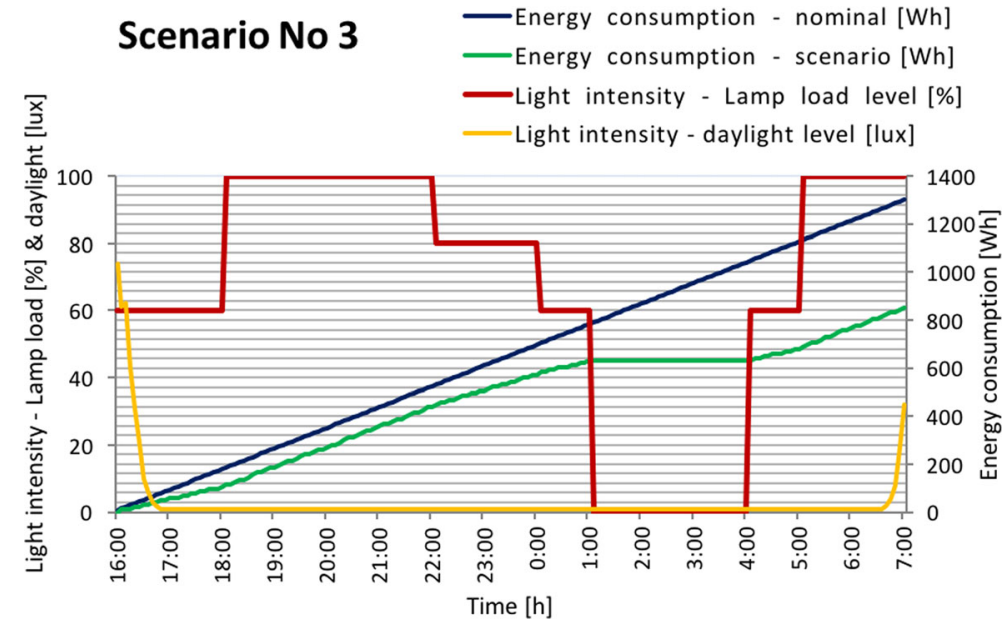


Fig. 13 Results of the energy consumption in the street lighting installation for a 1-year period
Energy consumption for 1 year period

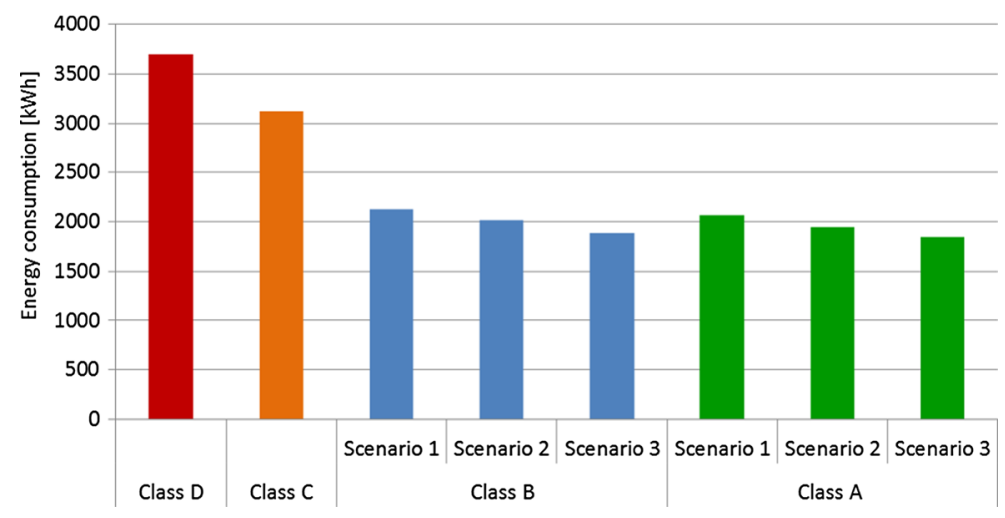

automation and control system assumptions (Figs. 8, 10, and 12) provide a little bit more electrical energy savings in the lighting system, compared to the functional strategy corresponded to the class B and of course to that without control functions (the class D and C). Those last are by about $45 \%$. It should be noticed that this result has been obtained from a set of real data from our experimental installation with the Lux sensor, presented in Fig. 2 and Fig. 4. A short summary of the results is presented in Table 1.

For the experimental street lighting installation corresponded to class A and for scenario 3, financial analysis has been carried out. Prices and costs are considered in it, arising from the Polish economic and political conditions. The analysis has established significant potential in operation cost savings. It is a direct result of the experimental street lighting system modernization from class D to class A. Finally, a payback time calculated for the mentioned case is 7 years. The return of investment (ROI) factor for the 2014 year is $12.5 \%$. Bearing in mind all these results, lighting systems realized in the class D (no control in practiceonly basic switch on/off manually or automatically by simple devices), should be modernized and new installations should no longer be built in such standard. The smallest power consumption characterized of course the lighting control systems made in the class A. However, it should be noted that they are more complex and require the involvement of a larger infrastructure (for instance additional sensors) than the class B systems. We propose to implement new, modern control systems of the street lighting in the public spaces in accordance with the class A objectives (EN 15232 Standard 2012). One of the ways to meet the mentioned communication and integration requirements is the use of the open, international standards and technologies of distributed network systems used in the BACS applications. The street lighting control systems are more and more

Table 1 The control scenarios, functional organization strategies classes and energy consumption — comparison

\begin{tabular}{|c|c|c|c|c|}
\hline Functional strategies class & Description & Scenarios & $\begin{array}{l}\text { Energy consumption } \\
\text { day }(15 \mathrm{~h}) \text { per lamp }\end{array}$ & $\begin{array}{l}\text { Energy consumption } 1 \text { year } \\
\text { for the whole lighting system }\end{array}$ \\
\hline \multirow[t]{3}{*}{ A } & \multirow{3}{*}{$\begin{array}{l}\text { Advanced integration; all kinds } \\
\text { of sensors and actuators }\end{array}$} & 1 A (Fig. 8) & $0.94 \mathrm{kWh}$ & $2061.92 \mathrm{kWh}$ \\
\hline & & 2 A (Fig. 10) & $0.89 \mathrm{kWh}$ & $1945.52 \mathrm{kWh}$ \\
\hline & & 3 A (Fig. 12) & $0.85 \mathrm{kWh}$ & $1844.88 \mathrm{kWh}$ \\
\hline \multirow[t]{3}{*}{$\mathrm{B}$} & \multirow{3}{*}{$\begin{array}{l}\text { Advanced integration; selected } \\
\text { sensors and actuators }\end{array}$} & $1 \mathrm{~B}$ (Fig. 7) & $0.97 \mathrm{kWh}$ & $2123.2 \mathrm{kWh}$ \\
\hline & & 2 B (Fig. 9) & $0.92 \mathrm{kWh}$ & $2012.48 \mathrm{kWh}$ \\
\hline & & 3 B (Fig. 11) & $0.87 \mathrm{kWh}$ & $1887.2 \mathrm{kWh}$ \\
\hline $\mathrm{C}$ & $\begin{array}{l}\text { Basic control functions; lamps } \\
\text { with electronic modules }\end{array}$ & C (Fig. 6) & $1.3 \mathrm{kWh}$ & $3117.6 \mathrm{kWh}$ \\
\hline $\mathrm{D}$ & $\begin{array}{l}\text { Without control; basic on/off } \\
\text { functions; lamps with } \\
\text { electromagnetic ballast }\end{array}$ & D (Fig. 6) & $1.54 \mathrm{kWh}$ & $3693.6 \mathrm{kWh}$ \\
\hline
\end{tabular}


popular in cities and they will be a standard element of their infrastructure in the future. They are and will be implemented in other applications as well: surrounding industrial plants, logistic centres, stadiums, entertainment centres etc. The ability of their integration with energy management systems (EMS) and the BMS is particularly attractive (Novak and Gerstinger 2010). In this sense, in our opinion, the research and experiments presented in this paper are very important. The results of our studies and experiments confirm that the integration with the BACS and the BMS along with proposed new approach to functional strategies organizing, based on the BACS efficiency classes, provide energy savings in the outdoor lighting systems. However, due to diverse and individual characteristics of the possible applications of public lighting (lighting highways, roads, streets, sidewalks, alleys, squares, parks etc.), the conclusions of the studies should be regarded as a case study and proposal of possible functional control algorithms or scheduling time.

\section{Conclusions}

In this paper new approach to infrastructure and control systems organization in the street and outdoor lighting installations is introduced. It is based on the EN-15,232 standard guidelines originally dedicated to organizing control functions and functional strategies in the BACS, but we propose a new concept to extending the application area of this standard in the street lighting installations. It has been decided since the control and monitoring functions available in the BACS and the TBM, provide tools to reduce the energy consumption in this kind of installations. Besides, the proposed approach to definition and formulation of the functional strategies provides additional value for scientists, engineers and integrators allowing them to organize more efficient outdoor lighting systems and to easy evaluate their potential energy consumption savings.

To verify this concept and impact of control systems organization on energy efficiency improvement, authors have proposed a set of functional strategies with respect to different BACS efficiency classes introduced in EN15,232 standard. They have been implemented in real street lighting installation. In the conducted experiments, we have confirmed that the BACS functions influence on the electrical energy consumption. In our study it has been proved that more advanced and well organized street lighting control systems could significantly improve the energy performance of the street lighting installations (results presented in Table 1 for the functional strategies corresponded to the class $\mathrm{B}$ and class A in comparison with class $\mathrm{C}$ and class D). The experimental results presented in Section 3 prove a potential and effectiveness of mentioned concept not only in this particular installation. Conclusions from analysis of results for these control systems organization could be generalized for other street and outdoor lighting installations, particularly with different light sources.

In comparison to state of the art of investigations concerning the exploration and development of lighting control algorithms, mentioned in the Introduction, our concept provides useful contribution to easier and more efficient control systems organization process. It is especially important for dynamic control systems using various infrastructure elements (sensors, actuators and advanced controllers). Results of our real experiment show that guidelines for the functional strategies corresponded to the Class A and Class B, proposed and verified in the paper, should be used in control systems organization process for street lighting installations. Moreover, the street lighting control systems with functional strategies corresponding to the Class D shall be retrofitted and new systems shall not be organized according to the Class $\mathrm{D}$ guidelines.

Furthermore, integration of the outdoor lighting systems into the BACS offer possibility to exchange information with other systems such as the Smart Metering - energy and media monitoring and other devices used in buildings as well. This allows to integrate the lighting of public spaces systems and other elements of the so-called the Smart Grids and the Smart Cities infrastructures.

\section{Future works}

Proposed approach and functional strategies based on the EN-15,232 standard could be implemented in other applications as well. One of the proposals for the future research and tests is an adaptive lighting. The aim of the research would be to ensure the safety of vehicles and people on the streets, car parks, squares etc., whereas reducing energy consumption and operating costs of the lighting systems. Hence, there is a need for continuous adjustment of the operating parameters to changing environmental conditions, resulting from changes in 
the intensity of daylight, variable traffic, changes in meteorological conditions and possible emergencies, unusual situations, etc. (Novak et al. 2013). Meeting these requirements can only be achieved by the integration of many system components and developing suitable algorithms for them, taking into account the dynamics of mentioned factors and parameters (VelascoQuesada et al. 2012; Wojnicki et al. 2014). Control systems with mixed communication technologies (e.g. PLC, wireless communication - IEEE 802.15.4) could be implemented in this kind of installations (Pantoni and Brandão 2011). Therefore, in next stage of our studies we are going to develop a predictive control algorithm, with ability to adapt to the changes of the seasons, months and schedules and even able to react to unexpected changes in the environmental parameters, etc. (Oezluek et al. 2010; Alzubaidi and Soori 2012; Lau et al. 2013; Novak et al. 2013). Another important question is the Internet protocol IPv6 and an Internet of Things (IoT) technologies growing. Authors are considering their implementation in the BACS in future works. The IoT devices with various types of wireless communication would be implemented and investigated in those kinds of implementations. This should create new possibilities of monitoring and control devices in the BMS and integrated the street lighting systems.

Open Access This article is distributed under the terms of the Creative Commons Attribution 4.0 International License (http:// creativecommons.org/licenses/by/4.0/), which permits unrestricted use, distribution, and reproduction in any medium, provided you give appropriate credit to the original author(s) and the source, provide a link to the Creative Commons license, and indicate if changes were made.

\section{References}

Alzubaidi S, Soori PK (2012) Study on energy efficient street lighting system design. In: 2012 I.E. International Power Engineering and Optimization Conference. IEEE, pp 291295.

Arnold, M., \& Barth, V. (2012). Open innovation in urban energy systems. Energy Efficiency, 5, 351-364. doi:10.1007 /s12053-011-9142-6.

Burgos-Payan M, Correa-Moreno F-J, Riquelme-Santos J-M (2012) Improving the energy efficiency of street lighting. A case in the South of Spain. In: 2012 9th International Conference on the European Energy Market. IEEE, pp 1-8.

SungKwan Cho, Dhingra V (2008) Street lighting control based on LonWorks power line communication. In: 2008 I.E. International Symposium on Power Line Communications and Its Applications. IEEE, pp 396-398.
Chung H.SH, Ho NM, Hui SYR, Mai WZ (2005) Case study of a highly-reliable dimmable road lighting system with intelligent remote control. In: 2005 European Conference on Power Electronics and Applications. IEEE, p 1 pp.-P.10.

Collotta, M., \& Tirrito, S. (2014). A flexible approach for smart management of transmissions in power line communications. J Comput Networks Commun, 2014, 1-9. doi: $10.1155 / 2014 / 393782$.

Colmenar-Santos, A., Terán de Lober, L. N., Borge-Diez, D., \& Castro-Gil, M. (2013). Solutions to reduce energy consumption in the management of large buildings. Energy and Buildings, 56, 66-77. doi:10.1016/j.enbuild.2012.10.004.

Corp, E. (2011). Echelon control network solutions save energy with smart street lighting in China. Echelon News, 1-4.

Djuric, N., \& Novakovic, V. (2010). Correlation between standards and the lifetime commissioning. Energy and Buildings, 42, 510-521. doi:10.1016/j.enbuild.2009.10.020.

Dragičević, T., Guerrero, J. M., Vasquez, J. C., Dragicevic, T., \& Guerrero, J. M. (2014). A distributed control strategy for coordination of an autonomous LVDC microgrid based on power-line signaling. IEEE Transactions on Industrial Electronics, 61, 3313-3326. doi:10.1109/TIE.2013.2282597.

EN 15232 Standard (2012) Energy performance of buildingsimpact of building automation, controls and building management

E-Street Project Grant Team (2008) Intelligent road and street lighting in Europe (E-Street)

European Commission (2013) Lighting the cities.

Fernandes, L. L., Lee, E. S., DiBartolomeo, D. L., \& McNeil, A. (2014). Monitored lighting energy savings from dimmable lighting controls in the New York Times Headquarters Building. Energy and Buildings, 68, 498514. doi:10.1016/j.enbuild.2013.10.009.

Gutierrez-Escolar, A., Castillo-Martinez, A., Gomez-Pulido, J. M., Gutierrez-Martinez, J.-M., González-Seco, E. P. D., \& Stapic, Z. (2016). A review of energy efficiency label of street lighting systems. Energy Efficiency. doi:10.1007/s12053-016-9454-7.

Hsieh, J.-C. C., \& Lin, J.-L. L. (2011). Novel single-stage selfoscillating dimmable electronic ballast with high power factor correction. IEEE Transactions on Industrial Electronics, 58, 250-262. doi:10.1109/TIE.2009.2017104.

Lau S.P, Merrett G V., White NM (2013) Energy-efficient street lighting through embedded adaptive intelligence. In: 2013 International Conference on Advanced Logistics and Transport. IEEE, pp 53-58.

Leccese, F. (2013). Remote-control system of high Ef fi ciency and intelligent street lighting using a ZigBee network of devices and sensors. IEEE Trans POWER Deliv, 28, 21-28. doi:10.1109/TPWRD.2012.2212215.

Li-jun Q, Zi-zheng S, Feng J (2011) Intelligent streetlight energysaving system based on LonWorks power line communication technology. In: 2011 4th International Conference on Electric Utility Deregulation and Restructuring and Power Technologies (DRPT). IEEE, pp 663-667.

Marcos Alonso, J., Ribas, J., Coz, J. J. D., Calleja, A. J., Alonso, J. M., Ribas, J., Member, S., Jos, J., Calleja, A. J., Member, A., Corominas, E. L., \& Rico-secades, M. (2000). Development of a distributive control scheme for fluorescent lighting based on LonWorks technology. IEEE Transactions on Industrial Electronics, 47, 1253-1262. doi:10.1109/41.887953. 
Mohamed, S. (2013). Smart street lighting control and monitoring system for electrical power saving by using VANET. International Journal of Communications, Network and System Sciences, 06, 351-360. doi:10.4236/ijens.2013.68038.

Moo, C.-S. S., Huang, C.-K. K., Lee, K.-H. H., \& Huang, D.-J. J. (2011). Repeatedly resonating ignition circuit for HID lamp electronic ballasts. IEEE Transactions on Industrial Electronics, 58, 244-249. doi:10.1109/TIE.2010.2044739.

Müllner, R., \& Riener, A. (2011). An energy efficient pedestrian aware smart street lighting system. Int J Pervasive Comput Commun, 7, 147-161. doi:10.1108/17427371111146437.

Noga M, Ożadowicz A, Grela J (2012) Modern, certified building automation laboratories AutBudNet-put "learning by doing" idea into practice. Electr Rev 137-141.

Noga, M., Ożadowicz, A., Grela, J., \& Hayduk, G. (2013). Active consumers in smart grid systems-applications of the building automation technologies. Electrical Review, 227-233.

Novak, T., \& Gerstinger, A. (2010). Safety- and security-critical services in building automation and control systems. IEEE Transactions on Industrial Electronics, 57, 3614-3621. doi:10.1109/TIE.2009.2028364.

Novak T, Pollhammer K, Zeilinger H, Schaat S (2014) Intelligent streetlight management in a smart city. In: Proceedings of the 2014 I.E. Emerging Technology and Factory Automation (ETFA). IEEE, pp 1-8

Novak T, Zeilinger H, Schaat S (2013) Increasing energy efficiency with traffic adapted intelligent streetlight management. In: IECON 2013 - 39th Annual Conference of the IEEE Industrial Electronics Society. IEEE, pp 6087-6092.

Oezluek AC, Ploennigs J, Kabitzsch K (2010) Designing building automation systems using evolutionary algorithms with semi-directed variations. In: 2010 I.E. International Conference on Systems, Man and Cybernetics. IEEE, pp 2328-2335.

Oliveira-Lima, J. A., Delgado-Gomes, V., Martins, J. F., \& Lima, C. (2014). Standard-based service-oriented infrastructure to integrate intelligent buildings in distributed generation and smart grids. Energy and Buildings, 76, 450-458. doi:10.1016/j.enbuild.2014.03.013.

Omar, M., Rahman, H., Majid, M., Rosmin, N., Hassan, M., \& Omar, W. W. (2013). Design and simulation of electronic ballast performance for high pressure sodium street lighting. Lighting Research and Technology, 45, 729-739. doi:10.1177/1477153512471365.

Onat, A., Naskali, T., Parlakay, E., \& Mutluer, O. (2011). Control over imperfect networks: model-based predictive networked control systems. IEEE Transactions on Industrial Electronics, 58, 905-913. doi:10.1109/TIE.2010.2051932.

Ożadowicz A, Grela J (2014) The street lighting integrated system case study, control scenarios, energy efficiency. In: 2014 I.E. Emerging Technology and Factory Automation (ETFA).

Palensky, P., \& Dietrich, D. (2011). Demand side management: demand response, intelligent energy systems, and smart loads. IEEE Trans Ind Informatics, 7, 381-388. doi:10.1109/TII.2011.2158841.

Pantoni, R., \& Brandão, D. (2011). A confirmation-based geocast routing algorithm for street lighting systems. Computers and Electrical Engineering, 37, 1147-1159. doi:10.1016/j. compeleceng.2011.06.004.

Pizzuti, S., Annunziato, M., \& Moretti, F. (2013). Smart street lighting management. Energy Efficiency, 6, 607-616. doi:10.1007/s 12053-013-9195-9.

Schiavon, S., \& Melikov, A. K. (2009). Energy-saving strategies with personalized ventilation in cold climates. Energy and Buildings, 41, 543-550. doi:10.1016/j. enbuild.2008.11.018.

Schönenberger, P. (2015). eu.bac System. Energy and Buildings, 100, 16-19. doi:10.1016/j.enbuild.2014.11.051.

Soucek, S., \& Sauter, T. (2004). Quality of service concerns in IPbased control systems. IEEE Transactions on Industrial Electronics, 51, 1249-1258. doi:10.1109/TIE.2004.837860.

Sutterlin P, Downey W (1998) A power line communication tutorial - challenges and technologies. In: International Symposium on Power Line Communications and Its Applications (ISPLC). pp 15-29.

Tascikaraoglu, A., Boynuegri, A. R., \& Uzunoglu, M. (2014). A demand side management strategy based on forecasting of residential renewable sources: a smart home system in Turkey. Energy and Buildings, 80, 309-320. doi:10.1016/j. enbuild.2014.05.042.

The European PPP Expertise Centre (EPEC) (2010) Energy Efficient Street Lighting: Guidelines.

Todorović, B. M., \& Samardžija, D. (2016). Road lighting energysaving system based on wireless sensor network. Energy Efficiency. doi:10.1007/s12053-016-9447-6.

Velasco-Quesada, G., Román-Lumbreras, M., \& Conesa-Roca, A. (2012). Comparison of central dimmer systems based on multiple-tapped autotransformer and high-frequency switching converter. IEEE Transactions on Industrial Electronics, 59, 1841-1848. doi:10.1109/TIE.2011.2112321.

Wojnicki, I., Ernst, S., Kotulski, L., \& Sedziwy, A. (2014). Advanced street lighting control. Expert Systems with Applications, 41, 999-1005. doi:10.1016/j.eswa.2013.07.044. 